\title{
Trellis Coded Spatial Modulation
}

\author{
Raed Mesleh, Member, IEEE, Marco Di Renzo, Member, IEEE, Harald Haas, Member, IEEE, \\ and Peter M. Grant, Fellow, IEEE
}

\begin{abstract}
Trellis coded modulation (TCM) is a well known scheme that reduces power requirements without any bandwidth expansion. In TCM, only certain sequences of successive constellation points are allowed (mapping by set partitioning). The novel idea in this paper is to apply the TCM concept to the antenna constellation points of spatial modulation (SM). The aim is to enhance SM performance in correlated channel conditions. SM considers the multiple transmit antennas as additional constellation points and maps a first part of a block of information bits to the transmit antenna indices. Therefore, spatial multiplexing gains are retained and spectral efficiency is boosted. The second part of the block of information bits is mapped to a complex symbol using conventional digital modulation schemes. At any particular time instant, only one antenna is active. The receiver estimates the transmitted symbol and the active antenna index and uses the two estimates to retrieve the original block of data bits. In this paper, TCM partitions the entire set of transmit antennas into sub-sets such that the spacing between antennas within a particular sub-set is maximized. The scheme is called trellis coded spatial modulation (TCSM). Tight analytical performance bounds over correlated fading channels are proposed in this paper. In addition, the performance and complexity of TCSM is compared to the performance of SM, coded V-BLAST (vertical Bell Labs layered space-time) applying near optimum sphere decoder algorithm, and Alamouti scheme combined with TCM. Also, the performance of all schemes with turbo coded modulation is presented. It is shown that under the same spectral efficiency, TCSM exhibits significant performance enhancements in the presence of realistic channel conditions such as Rician fading and spatial correlation (SC). In addition, the complexity of the proposed scheme is shown to be $80 \%$ less than the V-BLAST complexity.
\end{abstract}

Index Terms-Spatial modulation, MIMO, trellis coded modulation, V-BLAST, sphere decoder, space-time coding, Alamouti scheme, Rician fading, spatial correlation.

\section{INTRODUCTION}

$\mathbf{P}$ OWER and bandwidth are limited resources in wireless communication systems. While there have been significant enhancements in increasing system spectral efficiency with the advent of MIMO (multiple-input-multiple-output) techniques, these enhancements come at the cost of increased complexity which compromises power efficiency. Therefore,

Manuscript received March 18, 2009; revised February 14, 2010 and March 30, 2010; accepted April 11, 2010. The associate editor coordinating the review of this paper and approving it for publication was H. Ochiai.

R. Mesleh is with Jacobs University, Department of Electrical Engineering and Science, D-28759 Bremen, Germany (e-mail: r.mesleh@jacobsuniversity.de).

M. Di Renzo is with L2S, UMR 8506 CNRS - SUPELEC - Univ ParisSud, 3 rue Joliot-Curie, 91192 Gif-sur-Yvette CEDEX, France (e-mail: marco.direnzo@1ss.supelec.fr).

H. Haas and P. M. Grant are with the University of Edinburgh, Institute for Digital Communications, Joint Research Institute for Signal and Image Processing, the Kings Buildings, Edinburgh EH9 3JL, UK (e-mail: \{h.haas, Peter.Grant\}@ed.ac.uk).

Digital Object Identifier 10.1109/TWC.2010.07.091526 there is currently considerable interest in new technologies that allow for an efficient exploitation of both resources.

One very successful method of reducing power requirements without increasing bandwidth is trellis coded modulation (TCM) [1]. TCM is a modulation scheme which allows highly efficient and reliable digital transmission. TCM combines the function of convolutional encoder of rate $R=$ $r /(r+1)$, where $r$ is a positive integer number, and $M$-ary signal mapper that maps $M=2^{(r+1)}$ constellation points. Specifically, TCM uses $M$ constellation points to transmit at a rate of $r=\log _{2}\left(\frac{M}{2}\right)$ bits/symbol. As a result, a coding gain is obtained without any sacrifice in bandwidth. Unlike conventional coding techniques only certain sequences of successive constellation points are allowed (mapping by set partitioning). The key idea is to group symbols into sets of equal sizes where each set maximizes the distance between its symbols.

In addition to limited power and bandwidth resources, wireless communication system design has to deal with multipath propagation and interference. In the past, the ultimate goal of wireless communication was to combat the distortion caused by multipath in order to approach the theoretical limit of capacity for a band-limited channel. Spatial multiplexing MIMO constructively exploits multipath propagation to provide higher data throughput for the same given bandwidth [2]. In fact, multipath propagation can be considered as multiple channels between transmitter and receiver and these are used to transmit independent data streams.

In traditional MIMO systems, all transmit antennas are used at the same time to simultaneously transmit data to the receiver side. The aim is to improve power efficiency by maximizing spatial diversity (as in space-time coding (STC)) [3], [4], or to boost the data rate by transmitting independent streams from each transmit antenna (as in V-BLAST (vertical Bell Labs layered space-time)) [5], [6], or to achieve both of them at the same time at the expense of increasing complexity [7].

An alternative multiple antenna transmission technique, called spatial modulation (SM), utilizes the multiple transmit antennas in a different way [8], [9]. Multiple antennas are considered as additional constellation points that are used to carry information bits. At any one time instant, only one transmit antenna is active. The incoming bit sequence determines the active antenna index. The active antenna transmits the data symbol and both the transmitted symbol and the active antenna index are estimated at the receiver and used to decode the original information bits.

In this paper, the key idea of TCM is applied to the antenna constellation points of SM. The novel scheme is called trellis coded spatial modulation (TCSM). In TCSM, the transmit 


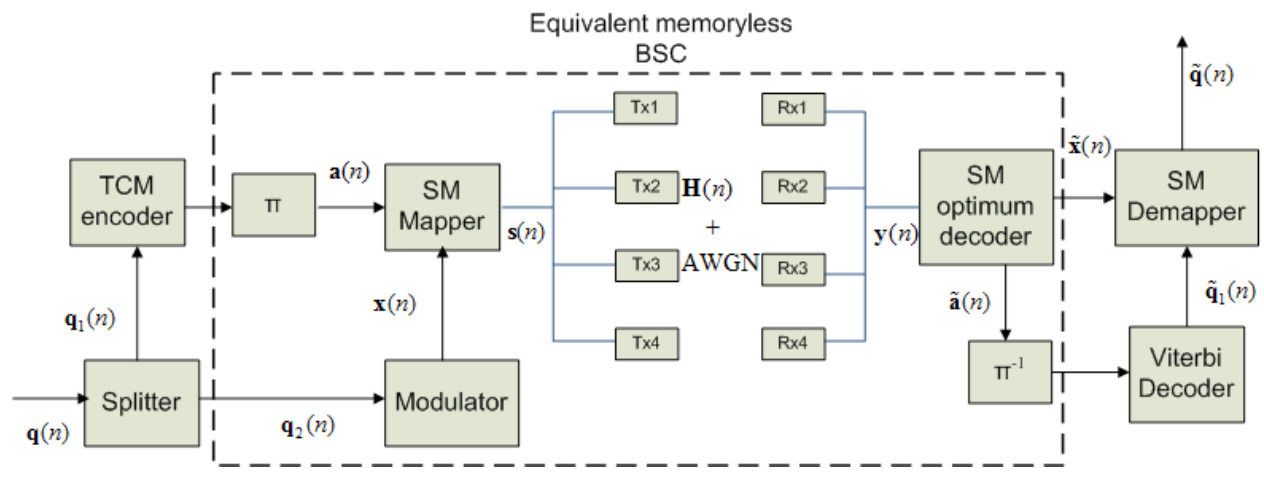

Fig. 1. Trellis coded spatial modulation (TCSM) system model.

antennas are partitioned into sub-sets, in such a way that the spatial spacing between antennas in the same sub-set is maximized. Therefore, the effect of correlated channels on the performance of SM is reduced. This is significant when considering portable devices with multiple antennas installed in compact space where sufficient element separation cannot be guaranteed. The performance of the proposed idea is analyzed in the presence of Rician fading and spatial correlation (SC) channels and a major bit error ratio (BER) improvement is reported as compared to SM, V-BLAST, and Alamouti scheme with the same spectral efficiency. V-BLAST with sphere decoder (SD) algorithm based on integer lattice theory proposed in [10], [11] and Alamouti scheme [12] combined with TCM and turbo coded modulation (TuCM) are considered.

In this paper, in addition, a simple but accurate analytical framework for performance analysis of TCSM over correlated fading channels is developed. For the sake of simplicity, the focus is on a MISO (multiple input single output) system with optimum detection and hard decision Viterbi decoding at the receiver. A tight upper bound for the average bit error probability (ABEP) is derived. Since in TCSM, convolutional (i.e., trellis) encoding is applied to transmit antenna indices only, it is assumed that each transmit antenna, when activated, transmits unmodulated data. This is equivalent to considering the application of trellis-based encoding to space shift keying (SSK) modulation [13]. SSK is a special case of SM where the active transmit antenna transmits unmodulated data and the data bits are encoded to spatial constellation points only.

The rest of the paper is organized as follows: In Section II, the system model of TCSM is presented. Channel models are discussed in Section III. Performance analyses of the proposed scheme are discussed in Section IV. The considered V-BLAST and Alamouti systems are discussed in Section V. Simulation results are presented in Section VI and the complexity comparison between TCSM, V-BLAST, and Alamouti systems are provided in Section VII. Finally, Section VIII concludes the paper.

\section{Trellis Coded Spatial Modulation (TCSM) SYSTEM MODEL}

The TCSM system model is depicted in Fig. 1. A MIMO system consisting of four transmit antennas $\left(N_{t}=4\right)$ and four receive antennas $\left(N_{r}=4\right)$ is considered as an example. The transmitted bits at each time instant are grouped as the row vectors of the matrix $\mathbf{q}(n)$. For illustration purposes, the incoming bit sequences are considered $\mathbf{q}(n)=\left[\begin{array}{ccc}101 & 110 & 011 \\ (n=1) & (n=2) & (n=3)\end{array}\right]^{T}$, where $(\cdot)^{T}$ denotes the transpose of a vector or a matrix. The first step is to split this matrix into two matrices. The first matrix $\mathbf{q}_{\mathbf{1}}(n)$ contains the bits that are mapped to spatial constellation points. While the second matrix contains the bits that are mapped to signal constellation points [8]. In the considered example, $\mathbf{q}_{\mathbf{1}}(n)=\left[\begin{array}{ccc}1 & 1 & 0 \\ (n=1) & (n=2) & (n=3)\end{array}\right]^{T}$ and $\mathbf{q}_{\mathbf{2}}(n)=$ $\left[\begin{array}{ccc}01 & 10 & 11 \\ (n=1) & (n=2) & (n=3)\end{array}\right]^{T}$. Assuming 4-PSK (phase shift keying) constellation and natural mapping, the second matrix is mapped to $\mathbf{x}(n)=\left[\begin{array}{ccc}j & -1 & -j \\ (n=1) & (n=2) & (n=3)\end{array}\right]^{T}$, where $j=\sqrt{-1}$ is the imaginary unit. Each element in this matrix corresponds to the symbol that is transmitted from one antenna among the set of existing transmit antennas at one discrete time instant. The first matrix, $\mathbf{q}_{\mathbf{1}}(n)$, is then used to select the active transmit antenna. However, before mapping the bits in the first matrix to the spatial constellation points (the transmit antenna indices), the bits are processed by a rate $1 / 2 \mathrm{TCM}$ encoder followed by a random block interleaver. The TCM encoder, state transition, and spatial mapping are depicted in Fig. 2. TCM groups the antenna indices in a tree like fashion, then separates them into two limbs of equal size. At each limb of the tree, the indices are further apart. In other words, TCM partitions the transmit antennas into subsets with the constraint of maximizing the spacing of antennas belonging to the same sub-set. In the given example and assuming all antennas are equally spaced on a straight line, antennas one and three form a set and antennas two and four form the other set as shown in Fig. 3. The output of the TCM encoder is then used to select the active antenna. In the above example, $\mathbf{q}_{\mathbf{1}}(n)$ is transformed into another matrix $\mathbf{a}(n)=\left[\begin{array}{ccc}01 & 11 & 11 \\ (n=1) & (n=2) & (n=3)\end{array}\right]^{T}$ by the encoder of Fig. 2(a) assuming the initial state of the encoder is 00 . The SM mapper operates on both $\mathbf{a}(n)$ and $\mathbf{x}(n)$ matrices creating the output matrix

$$
\mathbf{s}(n)=\left[\begin{array}{ccc}
0 & 0 & 0 \\
j & 0 & 0 \\
0 & 0 & 0 \\
0 & -1 & -j \\
(n=1) & (n=2) & (n=3)
\end{array}\right] .
$$




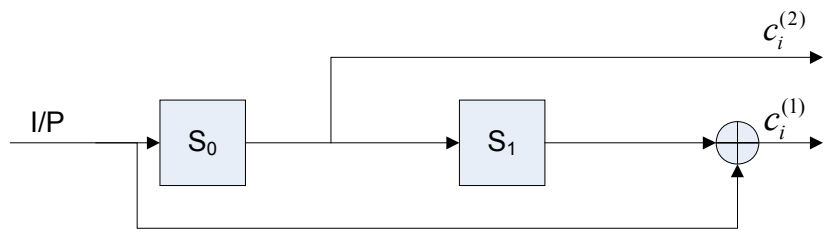

a) TCM Convolutional Encoder

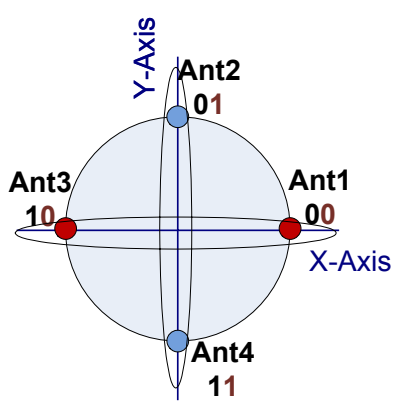

b) TCM encoded bits and their mapping to spatial constellation points

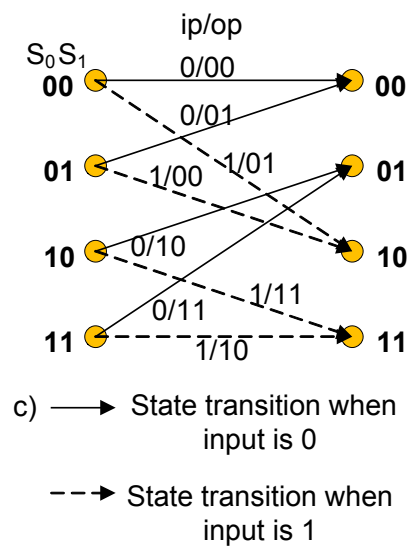

Fig. 2. Trellis coded encoder, spatial constellation mappings, and state transitions of the TCM encoder.

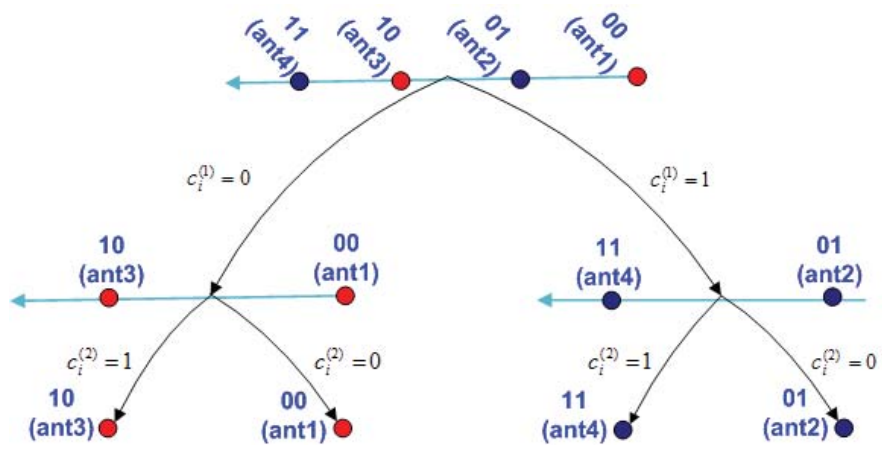

Fig. 3. TCSM spatial constellations set partitioning. In this figure, equal separation between the transmit antennas is assumed. TCSM partitions the antennas into sub-sets. Antennas one and three form a sub-set and antennas two and four form the other sub-set. Maximum separations between the antennas of each sub-set is achieved. $c_{i}^{(1)}$ and $c_{i}^{(2)}$ are the first and the second bit in the codeword $c_{i}$, respectively.

Each column from the output matrix is transmitted at a single discrete time instant from the existing transmit antennas over the MIMO channel $\mathbf{H}(n)$. For instance, at the first time instant in the considered example, the elements of the first column are transmitted from the four transmit antennas. Since, however, only one element is different from zero, only one antenna emits a signal. This means, that only the second antenna, $\ell=2$, is active at this particular time instant and is transmitting symbol $s_{\ell}=j$ while all other antennas are switched off. The signal experiences an $N_{r}$-dim additive white Gaussian noise (AWGN). The channel and the noise are assumed to have independent and identically distributed (i.i.d.) entries according to $\mathcal{C N}(0,1)$ and $\mathcal{C N}\left(0, \sigma_{n}^{2}\right)$, respectively; where $\sigma_{n}^{2}$ is the noise variance. In addition, Rician fading and Kronecker SC channel models are considered. The complete models for the channel are discussed in the next Section.

At the receiver, soft and hard decisions are considered.

\section{A. Hard decision}

The optimum SM decoder proposed in [14] is considered to estimate the transmitted symbol $\tilde{s}_{\ell}$ and the transmit antenna index $\tilde{\ell}$ as follows:

$$
\begin{aligned}
{\left[\tilde{s}_{\ell}, \tilde{\ell}\right] } & =\underset{\ell, s_{\ell}}{\arg \max } p_{\mathbf{y}}(\mathbf{y} \mid \mathbf{s}, \mathbf{H}) \\
& =\underset{\ell, s_{\ell}}{\arg \min } \sqrt{\rho}\left\|\mathbf{g}_{\ell}\right\|_{\mathrm{F}}^{2}-2 \operatorname{Re}\left\{\mathbf{y}^{H} \mathbf{g}_{\ell}\right\},
\end{aligned}
$$

where $\mathbf{g}_{\ell}=\mathbf{h}_{\ell} s_{\ell}$ is the received vector when transmitting the symbol $s_{\ell} \in\{\mathcal{S}=\{1: M\}\}$ from antenna index $\ell \in$ $\left\{\mathcal{L}=\left\{1: N_{t}\right\}\right\}$ where $\{\mathcal{S}\}$ and $\{\mathcal{L}\}$ are the set of signal constellation points and the set of spatial constellation points, respectively; $\mathbf{h}_{\ell}$ is the channel vector containing the path gains from transmit antenna $\ell$ to all receive antennas and $\operatorname{Re}(\cdot)$ is the real part of a complex number. In addition, $(\cdot)^{H}$ denotes the Hermitian of a vector or a matrix, $\rho$ is the average signal to noise ratio (SNR) at each receive antenna, and

$$
p_{\mathbf{y}}(\mathbf{y} \mid \mathbf{s}, \mathbf{H})=\pi^{-N_{t}} \exp \left(-\|\mathbf{y}-\sqrt{\rho} \mathbf{H} \mathbf{s}\|_{\mathrm{F}}^{2}\right)
$$

is the probability density function (pdf) of $\mathbf{y}$ conditioned on the transmitted vector $\mathbf{s}$ and the channel $\mathbf{H}$. The notation $\|\cdot\|_{\mathrm{F}}$ stands for the Frobenius norm of a vector or a matrix.

The estimated antenna index is de-mapped to the corresponding bits and the estimated data sequence of one complete frame, $\tilde{\mathbf{a}}(n)$, is applied to a random block deinterleaver and then decoded using a hard decision Viterbi decoder. The output from the Viterbi decoder together with the estimated symbols, $\tilde{\mathbf{x}}(n)$, are used to obtain the original information bits.

\section{B. Soft decision}

The data bits to be mapped to antenna indices and data symbols are jointly encoded by a turbo encoder. At the receiver, the log-likelihood ratios (LLRs) are first calculated and then processed by the turbo decoder. The computation of the LLRs is similar to the proposed SSK algorithm with TuCM in [13, eqn. (12)]. It is assumed that both, the bits encoded into the spatial domain and the bits encoded into the signal domain are independent and generated with equal probability due to the interleaver. The calculation of the LLRs for the $\imath^{\text {th }}$ spatial constellation bit is as follows,

$$
\begin{aligned}
L\left(\ell^{2}\right) & =\log \frac{P\left(\ell^{\imath}=1 \mid \mathbf{y}\right)}{P\left(\ell^{2}=0 \mid \mathbf{y}\right)} \\
& =\log \frac{\sum_{\tilde{\ell} \in \mathcal{L}_{1}^{2}} \sum_{s_{\ell} \in \mathcal{S}} p_{\mathbf{y}}(\mathbf{y} \mid \mathbf{s}, \mathbf{H}) P(\ell=\tilde{\ell})}{\sum_{\tilde{\ell} \in \mathcal{L}_{0}^{2}} \sum_{s_{\ell} \in \mathcal{S}} p_{\mathbf{y}}(\mathbf{y} \mid \mathbf{s}, \mathbf{H}) P(\ell=\tilde{\ell})} \\
& =\log \frac{\sum_{\tilde{\ell} \in \mathcal{L}_{1}^{2}} \sum_{s_{\ell} \in \mathcal{S}} \exp \left(-\frac{\left\|\mathbf{y}-\mathbf{h}_{\tilde{\ell}} \mathbf{s}_{\ell}\right\|^{2}}{\sigma_{n}^{2}}\right)}{\sum_{\tilde{\ell} \in \mathcal{L}_{0}^{2}} \sum_{s_{\ell} \in \mathcal{S}} \exp \left(-\frac{\left\|\mathbf{y}-\mathbf{h}_{\tilde{\ell}} \mathbf{s}_{\ell}\right\|^{2}}{\sigma_{n}^{2}}\right)}
\end{aligned}
$$

Similarly, the LLRs of the $j^{\text {th }}$ signal constellation bit is given as follows,

$$
L\left(s_{\ell}^{J}\right)=\log \frac{P\left(s_{\ell}^{J}=1 \mid \mathbf{y}\right)}{P\left(s_{\ell}^{J}=0 \mid \mathbf{y}\right)}
$$




$$
\begin{array}{r}
=\log \frac{\sum_{\tilde{s}_{\ell} \in \mathcal{S}_{1}^{\jmath}} \sum_{\tilde{\ell} \in \mathcal{L}} p_{\mathbf{y}}(\mathbf{y} \mid \mathbf{s}, \mathbf{H}) P\left(s_{\ell}=\tilde{s}_{\ell}\right)}{\sum_{\tilde{s}_{\ell} \in \mathcal{S}_{0}^{J}} \sum_{\tilde{\ell} \in \mathcal{L}} p_{\mathbf{y}}(\mathbf{y} \mid \mathbf{s}, \mathbf{H}) P\left(s_{\ell}=\tilde{s}_{\ell}\right)} \\
=\log \frac{\sum_{\tilde{s}_{\ell} \in \mathcal{S}_{1}^{J}} \sum_{\tilde{\ell} \in \mathcal{L}} \exp \left(-\frac{\left\|\mathbf{y}-\mathbf{h}_{\tilde{\ell}} \tilde{s}_{\ell}\right\|^{2}}{\sigma_{n}^{2}}\right)}{\sum_{\tilde{s}_{\ell} \in \mathcal{S}_{0}^{J}} \sum_{\tilde{\ell} \in \mathcal{L}} \exp \left(-\frac{\left\|\mathbf{y}-\mathbf{h}_{\tilde{\ell}} \tilde{s}_{\ell}\right\|^{2}}{\sigma_{n}^{2}}\right)}
\end{array}
$$

where $\mathcal{L}_{1}^{\imath}$ and $\mathcal{L}_{0}^{2}$ represent subsets from the set of spatial constellation points $\mathcal{L}$ which have "1" and " 0 " at the $\imath^{\text {th }}$ bit, respectively; $\mathcal{S}_{1}^{J}$ and $\mathcal{S}_{0}^{J}$ represent subsets from the set of signal constellation points $\mathcal{S}$ which have "1" and "0" at the $j^{\text {th }}$ bit, respectively.

\section{Channel Models}

In this paper, $\mathbf{H}$ is an $N_{r} \times N_{t}$ flat fading channel matrix representing the path gains $h_{i \nu}$ between transmit antenna $\nu$ and receive antenna $i$.

$$
\mathbf{H}=\left[\begin{array}{cccc}
h_{11} & h_{12} & \cdots & h_{1 N_{t}} \\
h_{21} & h_{22} & \cdots & h_{2 N_{t}} \\
\vdots & \vdots & \ddots & \vdots \\
h_{N_{r} 1} & h_{N_{r} 2} & \cdots & h_{N_{r} N_{t}}
\end{array}\right]
$$

In case of NLOS (non-line-of-sight), the sum of all scattered components of the received signal is modeled as a zero mean complex Gaussian random process given by $\alpha(t)=$ $\alpha_{1}(t)+j \alpha_{2}(t)$, where $\alpha_{1}(t)$ and $\alpha_{2}(t)$ are assumed to be real valued statistically independent Gaussian random processes. As a result, the phase of the random process $\alpha(t)$ takes a uniform distribution and the amplitude takes a Rayleigh distribution. Therefore, a static Rayleigh fading channel matrix that is flat for all frequency components is modeled.

\section{A. Rician fading channel}

If a LOS (line-of-sight) path exists between the transmit and receive antennas, the channel amplitude gain is characterized by a Rician distribution and the channel is said to exhibit Rician fading. The Rician fading MIMO channel matrix can be modeled as the sum of the fixed LOS matrix and a Rayleigh fading channel matrix as follows [15]:

$$
\mathbf{H}_{\text {Ricean }}=\sqrt{\frac{K}{1+K}} \overline{\mathbf{H}}+\sqrt{\frac{1}{1+K}} \mathbf{H},
$$

where $\sqrt{\frac{K}{1+K}} \overline{\mathbf{H}}$ is the LOS component, $\sqrt{\frac{1}{1+K}} \mathbf{H}$ is the fading component, and $K$ is the Rician $K$-factor. The Rician $K$-factor is defined as the ratio of the LOS and the scatter power components and $\overline{\mathbf{H}}$ is a matrix with all elements being one.

\section{B. Spatial correlation (Kronecker model)}

The channel correlation depends on both the environment and the spacing of the antenna elements. It is assumed that correlations at the transmitter and receiver array are independent of each other because the distance between the transmit and receive array is large compared to the antenna element spacing.
To incorporate the SC into the channel model, the correlation among channels at multiple elements need to be calculated. The cross correlation $\varphi_{\kappa \nu}$ between the channel coefficients of the two transmit antenna elements $(\kappa, \nu)$, with $\kappa, \nu=1,2, \ldots, N_{t}$, can be calculated as

$$
\varphi_{\kappa \nu}=\mathrm{E}\left\{\mathbf{h}_{\kappa} \mathbf{h}_{\nu}^{H}\right\}
$$

where $\mathrm{E}(\cdot)$ is the expectation operator, $\mathbf{h}_{\kappa}$ and $\mathbf{h}_{\nu}$ denote column vector $\kappa$ and column vector $\nu$ from the channel matrix $\mathbf{H}$, respectively.

These correlation coefficients are used to construct the correlation matrix at the transmitter, $\mathbf{R}_{\mathrm{tx}}$, as follows:

$$
\mathbf{R}_{\mathrm{tx}}=\left(\begin{array}{cccc}
\varphi_{11} & \varphi_{12} & \cdots & \varphi_{1 N_{t}} \\
\varphi_{21} & \varphi_{22} & \cdots & \varphi_{2 N_{t}} \\
\vdots & \vdots & \ddots & \vdots \\
\varphi_{N_{t} 1} & \varphi_{N_{t} 2} & \cdots & \varphi_{N_{t} N_{t}}
\end{array}\right)
$$

In a similar way, the correlation matrix at the receiver can be computed.

The correlated channel matrix is then modeled using the Kronecker model [16],

$$
\mathbf{H}^{\mathrm{corr}}=\frac{1}{\sqrt{\operatorname{tr}\left(\mathbf{R}_{\mathrm{rx}}\right)}} \mathbf{R}_{\mathrm{rx}}^{1 / 2} \mathbf{H} \mathbf{R}_{\mathrm{tx}}^{1 / 2}
$$

where $\operatorname{tr}(\cdot)$ denotes the trace of a matrix.

The correlation matrices can be computed analytically based on the power azimuth spectrum (PAS) distribution and array geometry [16]. A clustered channel model, in which groups of scatterers are modeled as clusters located around the transmit and receive antennas, is assumed. The calculation of the correlation matrices is discussed in details in [17, Sec. 2.2.3] and omitted here for the sake of brevity. The clustered channel model is validated through measurements [18] and adopted by various wireless system standard bodies such as the IEEE 802.11n Technical Group (TG) [19] and the 3GPP/3GPP2 Technical Specification Group (TSG) [20].

In this paper, similar parameters as discussed in [8] are adopted here as well except that the element spacing at the transmitter and the receiver are set to $0.1 \lambda$ and $0.5 \lambda$, respectively; where $\lambda$ is the wavelength. The $0.5 \lambda$ separation between the antennas can achieve relatively low correlation assuming the receiver is surrounded by a large number of local scatterers [21]. The $0.1 \lambda$ element spacing models a small mobile device with multiple antennas where a large separation between the antennas cannot be achieved.

\section{TCSM PERFormanCE ANALYSiS}

The analytical calculation of TCSM with more than one receive antenna is very complicated especially when considering channel correlation. Also, in TCSM, the bits to be mapped to transmit antenna indices are encoded while the other bits, which are mapped to signal constellations, are uncoded as discussed in Section II. An analytical treatment of the jointly coded system (in the spatial and the signal domain) is beyond the scope of this paper since the main contribution is the application of trellis coding in the spatial domain where the latter is enabled by the concept of spatial modulation. Therefore and in order to simplify the analysis, an $N_{t} \times 1$ 
MISO system is assumed where each transmit antenna, when activated, transmits unmodulated data similar to SSK [13].

The SC model for the analytical analysis assumes no correlation between the real and imaginary parts of the channel coefficients, i.e. $\mathrm{E}\left\{\left(h_{\kappa}^{\mathrm{R}}\right)\left(h_{\nu}^{\mathrm{I}}\right)\right\}=0 \forall \kappa, \nu$, where $h_{\kappa}=$ $h_{\kappa}^{\mathrm{R}}+j h_{\kappa}^{\mathrm{I}}$ is the channel gain from transmit antenna $\kappa$ to the receive antenna with superscripts $(\cdot)^{\mathrm{R}}$ and $(\cdot)^{\mathrm{I}}$ denoting the real and imaginary parts of complex number, respectively; $h_{\nu}=h_{\nu}^{\mathrm{R}}+j h_{\nu}^{\mathrm{I}}$ is the channel gain from transmit antenna $\nu$ to the receive antenna, and $h_{\nu}^{\mathrm{R}} \sim \mathcal{N}\left(0, \sigma_{h}^{2}\right), h_{\nu}^{\mathrm{I}} \sim \mathcal{N}\left(0, \sigma_{h}^{2}\right)$, $h_{\kappa}^{\mathrm{R}} \sim \mathcal{N}\left(0, \sigma_{k}^{2}\right)$, and $h_{\kappa}^{\mathrm{I}} \sim \mathcal{N}\left(0, \sigma_{k}^{2}\right)$, with $\sim \mathcal{N}\left(\mu, \sigma^{2}\right)$ denoting a normal random variable (RV) with mean $\mu$ and variance $\sigma^{2}$. The correlation between two channel coefficients is then given by $\varphi_{\kappa \nu}=\mathrm{E}\left\{\left(h_{\kappa}^{\mathrm{R}}\right)\left(h_{\nu}^{\mathrm{R}}\right)\right\}=\mathrm{E}\left\{\left(h_{\kappa}^{\mathrm{I}}\right)\left(h_{\nu}^{\mathrm{I}}\right)\right\} \forall \kappa, \nu$. Despite the limiting nature of these assumptions, they enable us to obtain insightful and simple analytical results for the complex system while still guaranteeing good adherence to physical reality.

\section{A. Methodology}

For analytical tractability, an infinite interleaving depth is assumed; thereby resulting in an ideal memoryless channel. In practice, the interleaver depth is finite and chosen in relation to the anticipated maximum fade duration. The methodology used for performance analysis assumes that the block diagram in Fig. 1 can be readily shown to be equivalent to a memoryless binary symmetric channel (BSC) whose inputs are the outputs of a generic convolutional encoder, and whose outputs are the inputs of a maximum-likelihood sequence estimation (MLSE) decoder, which implements the hard-decision Viterbi algorithm as shown in Fig. 1. As a consequence, the highlighted TCSM system model shown in Fig. 1 reduces to the equivalent communication system in, e.g., [22, Fig. 10].

In light of the above equivalence with the BSC, the ABEP of TCSM can be computed by resorting to the general theory for performance analysis of convolutional codes over memoryless BSCs [22, Sec. VII-A], which, however, needs to be specialized to the specific signal structure of TCSM modulation scheme over correlated fading channels.

\section{B. ABEP of convolutional codes over BSCs}

A general approach for the analysis of the performance of convolutional codes over memoryless channels is described in [22] in a comprehensive fashion. In particular, for BSCs the ABEP for MLSE detection can be computed either using the simple Chernoff bound (CB) or the true union bound (TUB), as summarized in what follows.

1) Chernoff bound: The CB for the ABEP of TCSM is as follows [22, eqn. (21)]:

$$
\mathrm{ABEP} \leq \mathrm{ABEP}_{\mathrm{CB}}=\left.\frac{d T(D, N)}{d N}\right|_{N=1, D=2 \sqrt{p_{\mathrm{BSC}}\left(1-p_{\mathrm{BSC}}\right)}}
$$

where $p_{\mathrm{BSC}}$ is the average error probability of the equivalent BSC channel and $T(D, N)$ is the transfer function of the augmented state diagram of the convolutional encoder used at the transmitter.
2) True union bound: The TUB for the ABEP of TCSM is as follows [22, eqn. (20)]:

$$
\mathrm{ABEP} \leq \mathrm{ABEP}_{\mathrm{TUB}}=\sum_{k=d_{\text {free }}}^{+\infty} q_{k} Z_{k}
$$

with $d_{\text {free }}$ denotes the free distance of the convolutional code and $Z_{k}$ being defined in (12), and $q_{k}$ being weighting coefficients that can be obtained from $T(D, N)$ as follows:

$$
\left.\frac{d T(D, N)}{d N}\right|_{N=1}=\sum_{k=d_{\mathrm{free}}}^{+\infty} q_{k} D^{k}
$$

According to (10)-(13), both CB and TUB can be computed when both $p_{\mathrm{BSC}}$ and $T(D, N)$ are known in closed-form. In particular, $p_{\mathrm{BSC}}$ depends on the specific transmission technology used to convey the information from the transmitter to the receiver. On the other hand, $T(D, N)$ depends on the particular trellis encoder used at the transmitter. Tight bounds for computing $p_{\mathrm{BSC}}$ over correlated Rayleigh fading channels and an arbitrary number of transmit antennas will be discussed next.

\section{Bounds for computing $p_{\mathrm{BSC}}$}

In the considered analysis, the $p_{\mathrm{BSC}}$ is the ABEP of an uncoded $N_{t} \times 1$ MISO system with SSK modulation at the transmitter and ML detection at the receiver [14]. Performance bounds for computing the ABEP of uncoded SSK modulation can be found in [13] for uncorrelated Rayleigh fading and an arbitrary number of transmit and receive antennas. However, these bounds have two main limitations: i) They are relatively weak, and ii) they are semi-analytic and require the estimation of the effective constellation points of SSK. Moreover, they have been derived and analyzed for uncorrelated fading. In what follows, two new upper bounds for performance prediction are proposed.

1) Symbol-based union bound for $p_{\mathrm{BSC}}$ : The first bound, which is called symbol-based union bound (SUB), can be obtained by using typical methods for performance analysis of multi-level modulation schemes with optimum detection [23, Sec. 5.7]. In particular, the average error probability for the antenna indices, i.e., the average constellation error probability (ACEP), can be readily upper bounded via union bound techniques, as follows [23, eqn. (5.86)]:

$$
\mathrm{ACEP} \leq \frac{1}{N_{t}} \sum_{\kappa=1}^{N_{t}} \sum_{\nu \neq \kappa=1}^{N_{t}} \operatorname{PEP}(\kappa \rightarrow \nu)
$$

where $\operatorname{PEP}(\kappa \rightarrow \nu)$ denotes the pairwise error probability (PEP) between the transmit antenna indices $\kappa$ and $\nu$, i.e., the probability of detecting that antenna index $\nu$ is active when, instead, antenna index $\kappa$ is actually active.

From the ACEP in (14), an upper bound, $p_{\mathrm{BSC}}^{\mathrm{SUB}}$, for $p_{\mathrm{BSC}}$ can be obtained by using [23, eqn. (5.101)], which assumes that the errors for all antenna indices are equally likely, as follows:

$$
p_{\mathrm{BSC}} \leq p_{\mathrm{BSC}}^{\mathrm{SUB}}=\frac{N_{t} / 2}{N_{t}-1} \mathrm{ACEP}
$$

2) Codeword-based union bound for $p_{\mathrm{BSC}}$ : The second bound, which is called codeword-based union bound (CUB), 


$$
Z_{k}= \begin{cases}\sum_{e=(k+1) / 2}^{k}\left(\begin{array}{l}
k \\
e
\end{array}\right) p_{\mathrm{BSC}}^{e}\left(1-p_{\mathrm{BSC}}\right)^{k-e}, & k \text { odd } \\
\frac{1}{2}\left(\begin{array}{c}
k \\
k / 2
\end{array}\right) p_{\mathrm{BSC}}^{k / 2}\left(1-p_{\mathrm{BSC}}\right)^{k / 2}+\sum_{e=(k / 2)+1}^{k}\left(\begin{array}{l}
k \\
e
\end{array}\right) p_{\mathrm{BSC}}^{e}\left(1-p_{\mathrm{BSC}}\right)^{k-e}, & k \text { even }\end{cases}
$$

can be obtained by using typical methods for performance analysis of MLSE detectors [24, Sec. 13.1.3]. In particular, an upper bound, $p_{\mathrm{BSC}}^{\mathrm{CUB}}$, for $p_{\mathrm{BSC}}$ can be obtained from $[24, \mathrm{Sec}$. $13.44]$, as shown in what follows $\left(p_{\mathrm{BSC}} \leq p_{\mathrm{BSC}}^{\mathrm{CUB}}\right)$ :

$$
p_{\mathrm{BSC}}^{\mathrm{CUB}}=\frac{1}{\log _{2}\left(N_{t}\right)} \frac{1}{N_{t}} \sum_{\kappa=1}^{N_{t}} \sum_{\nu \neq \kappa=1}^{N_{t}} \mathbb{N}(\kappa, \nu) \operatorname{PEP}(\kappa \rightarrow \nu)
$$

where $\mathbb{N}(\kappa, \nu)$ is the number of information bits errors committed by choosing $\kappa$ instead of $\nu$ as transmit antenna index and the following facts are considered: i) in an uncoded system the number of information bits per transmission is $\log _{2}\left(N_{t}\right)$, and ii) due to the interleaver, the antenna indices are transmitted with equal probability, which is equal to $1 / N_{t}$.

3) Computation of $\{\operatorname{PEP}(\kappa \rightarrow \nu)\}_{\kappa \neq \nu=1}^{N_{t}}$ : Both bounds in (15) and (16) need a closed-form expression for $\{\operatorname{PEP}(\kappa \rightarrow \nu)\}_{\kappa \neq \nu=1}^{N_{t}}$ to be computed in closed-form. For uncorrelated Rayleigh fading channels the PEP can be found in [13], while for correlated Nakagami- $m$ fading channels a general framework has been recently proposed in [25]. In particular, the PEP conditioned onto fading channel statistics can be written as follows [13], [25], [26]:

$$
\operatorname{PEP}\left(\kappa \rightarrow \nu \mid h_{\kappa}, h_{\nu}\right)=Q\left(\sqrt{\bar{\gamma}\left|h_{\kappa}-h_{\nu}\right|^{2}}\right)
$$

where $Q(x)=(1 / \sqrt{2 \pi}) \int_{x}^{+\infty} \exp \left(-t^{2} / 2\right) d t$ is the Qfunction and $\bar{\gamma}=E_{n} /\left(4 N_{0}\right)$. The notation $E_{n}$ is the average energy transmitted by each antenna that emits a non-zero signal, $|\cdot|$ denotes the absolute value, and $N_{0}$ is the doublesided power spectral density for the real and imaginary parts of the AWGN.

By following the same methodology described in [25], and after a few straightforward algebraic manipulations as shown in Appendix A, the PEP is given as follows:

$$
\begin{aligned}
\operatorname{PEP}(\kappa \rightarrow \nu) & =\operatorname{E}\left\{\operatorname{PEP}\left(\kappa \rightarrow \nu \mid h_{\kappa}, h_{\nu}\right)\right\} \\
& =\frac{1}{2}-\frac{1}{2} \sqrt{\frac{\bar{\sigma}_{\nu, \kappa}^{2} \bar{\gamma}}{1+\bar{\sigma}_{\nu, \kappa}^{2} \bar{\gamma}}}
\end{aligned}
$$

where $\bar{\sigma}_{\nu, \kappa}^{2}=\sigma_{\nu}^{2}+\sigma_{\kappa}^{2}-2 \varphi_{\nu, \kappa} \sigma_{\nu} \sigma_{\kappa}$ and $\operatorname{PEP}(\kappa \rightarrow \nu \mid A, B)$ is the PEP between the transmit antenna indices $\kappa$ and $\nu$ conditioned on RVs $A$ and $B$.

\section{Conventional Approaches}

\section{A. V-BLAST system model}

One of the most promising MIMO techniques to achieve the expected high data rate is the proposed V-BLAST architecture [6]. In V-BLAST, the information bit stream is separated in substreams. All the symbols of a certain stream are transmitted through the same antenna (one stream per antenna). The substreams are co-channel signals, that is, they have the same frequency band. Therefore, as compared to a SISO system, a linear increase of the data rate with the number of transmit antennas is achieved. The major task at the receiver is to resolve the inter-channel-interference (ICI) between the transmitted symbols. There are several detection algorithms available in the literature for V-BLAST. The optimum solution is to use maximum likelihood (ML) decoder. The problem of ML algorithm is the high complexity required to search over all possible combinations. Therefore, other algorithms are proposed trying to achieve similar performance as ML detection but with a significant reduction in receiver complexity. One of the most viable techniques is the proposed sphere decoder (SD) algorithm in [10], [11]. The main idea behind SD is to limit the number of possible codewords by considering only those codewords that are within a sphere centered at the received signal vector. The complexity of separating these signals should be small enough such that the overall complexity of the sphere decoding is lower than that of the full search.

The considered V-BLAST system in this paper is depicted in Fig. 4. The rate 1/2 convolutional encoder shown in Fig. 2 is considered. Coded V-BLAST system is generally termed horizontal BLAST (H-BLAST) [27]. In H-BLAST, the incoming bit stream is demultiplexed into $N_{t}$ parallel substreams. Channel coding followed by interleaving is applied to each substream. The coded bits are modulated and then transmitted from the corresponding transmit antenna. If the interleaving depth is selected to be larger than the coherence time of the channel, additional diversity gain can be achieved [28].

Another way of applying channel coding to V-BLAST is to use a single channel code for all layers as shown in Fig. 4. This scheme is called single coded BLAST (SCBLAST) [28]. SCBLAST is simpler than H-BLAST in the sense that only a single channel encoder is needed for all layers. In addition, in correlated slow or block fading channel, SCBLAST outperforms H-BLAST since the demultiplexer (at the transmitter) and multiplexer (at the receiver) act as spatial interleavers where they together help in breaking some of the correlation in the received signal [28]. For fair comparison with the proposed scheme, H-BLAST and SCBLAST performances in the different channel conditions considered in this paper are simulated and the results show an almost identical match. Therefore, SCBLAST is considered for the comparisons used in this paper due to its low complexity.

At the receiver, SD algorithm is employed to detect the transmitted symbols from all layers. In simulations, SD algorithm based on integer lattice theory is implemented. A complex MIMO system is decoupled into its real and imaginary parts so as to form an equivalent real-valued system. This approach is most appropriate for lattice-based modulation schemes such as quadrature amplitude modulation (QAM) or pulse amplitude modulation (PAM) [10], [11], [29]. For 

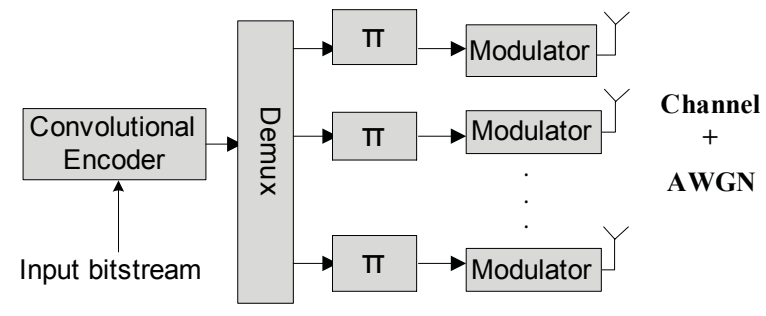

Fig. 4. V-BLAST system model.

other complex constellations such as phase-shift keying (PSK), the SD based on integer lattice theory are inefficient due to the existence of invalid candidates. A solution is to avoid decoupling of the complex system by applying complex SD algorithms [30].

The SD algorithm avoids an exhaustive search by examining only those points that lie inside a sphere with radius $C$. The performance of the SD algorithm is closely tied to the choice of the initial radius. The radius should be chosen large enough so that the sphere contains the solution. However, the larger the radius is, the longer the search takes which increases the complexity. On the other hand, a small radius may cause the algorithm to fail finding any point inside the sphere. In this paper, the initial radius of the SD algorithm is adjusted according to the noise level assuming the knowledge of the SNR at the receiver side. If no point is found inside the sphere, the search is repeated with a larger radius $(C=C+1)$ [31]. This approach is shown to perform near optimum maximum likelihood detection [31].

SD receivers have been implemented in custom applicationspecific integrated circuits (ASICs) [32] and as simplified fixed complexity designs [33] conveniently realized in fieldprogrammable gate arrays (FPGAs) [34].

The output symbols from SD are demodulated and the bits are deinterleaved. The bits from all layers are multiplexed and hard decision Viterbi decoder is then applied.

\section{B. Alamouti system model}

STC and in particular, Alamouti scheme, are widely considered and has been adopted in several wireless standards such as CDMA2000, UMTS (universal mobile telecommunications system), and IEEE802.16 [35]. The wide popularity of the Alamouti scheme is mainly driven by the high diversity gain, low complexity [36], and the robustness to realistic channel impairments such as Rician fading and SC [37], [38]. The considered Alamouti system model in this paper is depicted in Fig. 5. Two transmit antennas and four receive antennas are considered. Similar number of received antennas is considered in SCBLAST and TCSM. However, the modulation order is varied to achieve the target spectral efficiency. In addition, a maximum of four transmit antennas are considered in all compared systems. Generally, it is accepted that a large number of transmit antennas is impractical with current technology, especially when considering the cost that comes from adding antennas for an end-user system. For instance, two competing approaches are considered in the MIMO-oriented version of the IEEE 802.11n standard: one with a $2 \times 2$ MIMO matrix, and the other with a $4 \times 4$ matrix [39].

Alamouti scheme combined with TCM is considered [12]. The incoming bit sequence is first encoded by a rate $4 / 5 \mathrm{TCM}$ encoder as shown in Fig. 5. The output bits are modulated using 32QAM modulator. The Alamouti encoder takes two constellation symbols $\left(x_{1}\right.$ and $\left.x_{2}\right)$ and generates the Alamouti block code $\mathbf{s}=\left[\begin{array}{cc}x_{1} & x_{2} \\ -x_{2}^{*} & x_{1}^{*}\end{array}\right]$ [3]. The resulting block of signals is transmitted at two time instants from the two transmitting antennas. The channel is assumed to be quasistatic over the Alamouti codeword period. At the receiver, the transmitted symbols are estimated as follows [3], [40],

$$
\begin{aligned}
& \tilde{x}_{1}=\sum_{i=1}^{N_{r}} y_{1 i} h_{i 1}^{*}+y_{2 i}^{*} h_{i 2} \\
& \tilde{x}_{2}=\sum_{i=1}^{N_{r}} y_{1 i} h_{i 2}^{*}-y_{2 i}^{*} h_{i 1},
\end{aligned}
$$

where $y_{1 i}$ and $y_{2 i}$ are the received data at receive antenna number $i$ for two consecutive time instants. The decoded symbols are demodulated and the output bits for each transmitted frame are decoded using a hard decision Viterbi decoder.

\section{RESULTS}

\section{A. Analytical framework validation results}

For the analytical analysis, a $4 \times 1$ TCSM MISO system is considered. The calculation of the derived bounds in (10) and (11) requires the knowledge of the transfer function of the convolutional encoder. In this paper, a rate $1 / 2$ convolutional encoder with augmented transfer function $T(D, N)$ taken from [22, Fig. 1] is used. The encoder has an octal representation of $(5,7)$, constraint length of 3 , free distance of $d_{\text {free }}=5$, and $T(D, N)$ given by

$$
T(D, N)=\frac{N D^{5}}{1-2 N D}=\sum_{k=5}^{+\infty} 2^{k-5} N^{k-4} D^{k}
$$

The correlation between the transmit antennas $\kappa, \nu$ is defined as:

$$
\left\{\varphi_{\kappa \nu}\right\}_{\kappa, \nu=1}^{N_{t}}=\exp (-|\kappa-\nu| / 2) .
$$

Two cases are studied in the analysis: balanced and unbalanced setups. In the former case $\left\{\sigma_{i}^{2}\right\}_{i=1}^{N_{t}}=1$, while in latter case $\left\{\sigma_{i}^{2}\right\}_{i=1}^{N_{t}}$ are assumed to be independent and uniformly distributed (one-shot realization) in [8], [41]. For the Monte Carlo simulations, 1000 bit error events for estimating the 


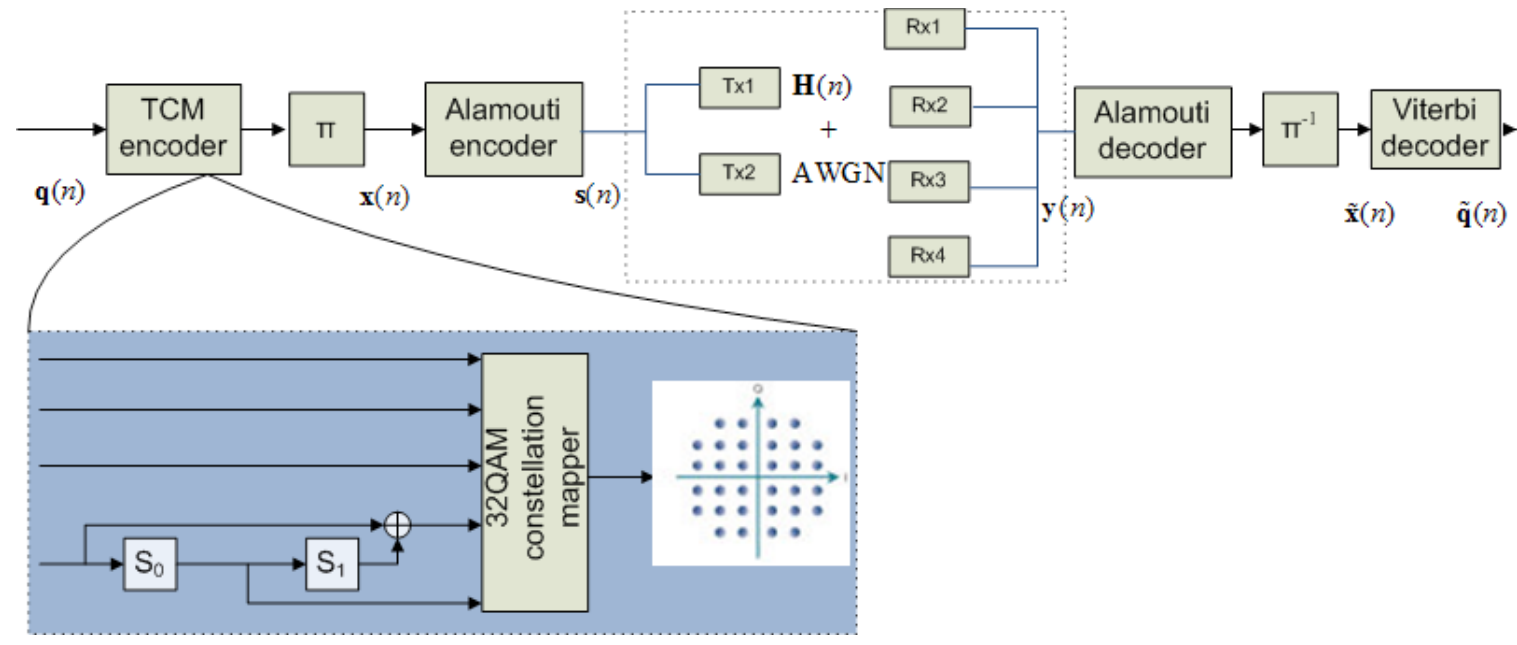

Fig. 5. Alamouti combined with TCM system model.

ABEP are required. In addition, the interleaver depth is set to 1000 and the traceback depth is set to 15 . As far as the computation of (11) is considered, the infinite series after the first 10 nonzero terms is truncated.

In the first results depicted in Fig. 6, the average error probability of the equivalent $\mathrm{BSC}$ channel, $p_{\mathrm{BSC}}$, is shown and compared with the bounds proposed in Section IV-C, along with the bound proposed in [13, eqn. (4)]. A very good accuracy of the proposed frameworks can be observed in the figure. The bound introduced in [13] overestimates the simulated $p_{\mathrm{BSC}}$ of approximately $2 \mathrm{~dB}$. On the contrary, both bounds proposed in this paper are asymptotically tight for high (but pragmatic) SNRs and yield almost similar performance to Monte Carlo results. The main reasons why the bounds in this paper are tighter than the bound proposed in [13] can be explained as follows: First, the authors in [13] themselves show that their bound can be very weak and that, in general, it is not asymptotically tight. Second, to overcome this problem, an ad hoc bound is proposed for Rayleigh fading channels [13, eqn. (9)], where a correction term is introduced to obtain the desired accuracy for large SNRs. A closed-form expression for this latter correction term is not given in [13]. The correction term has to be computed for each number of transmitantennas and fading distributions. The proposed bounds in this paper offer a simple way to overcome this problem by avoiding the ad hoc approach in [13, eqn. (4)]. In fact, in this paper we have demonstrated that it is indeed reasonable to apply a constant correction factor to the bound in [13]. More precisely, the additional constant correction factor is found to be $1 / \log _{2}\left(N_{t}\right)$.

Analytical and simulation results for coded system (TCSM) are depicted in Fig. 7. The ABEP of TCSM is shown and the proposed bounds are compared to each other and with Monte Carlo simulations. The error probability of the equivalent BSC is computed by using $p_{\mathrm{BSC}}^{\mathrm{SUB}}$. Both the $\mathrm{CB}$ in (10) and the TUB in (11) can accurately capture the diversity order of the system, i.e., the slope of the ABEP for high SNRs. However, the CB is rather weak and errors of around $5 \mathrm{~dB}$ can be observed in the analyzed system setup. On the other hand, the TUB is fairly accurate and asymptotically tight for high SNRs. These results
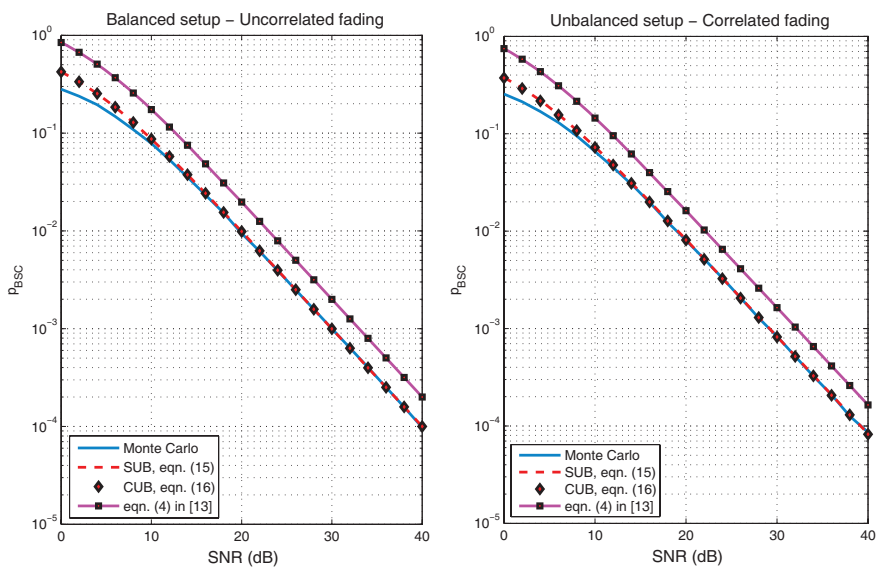

Fig. 6. $p_{\mathrm{BSC}}$ for a $4 \times 1$ MISO system: simulation vs. bounds.
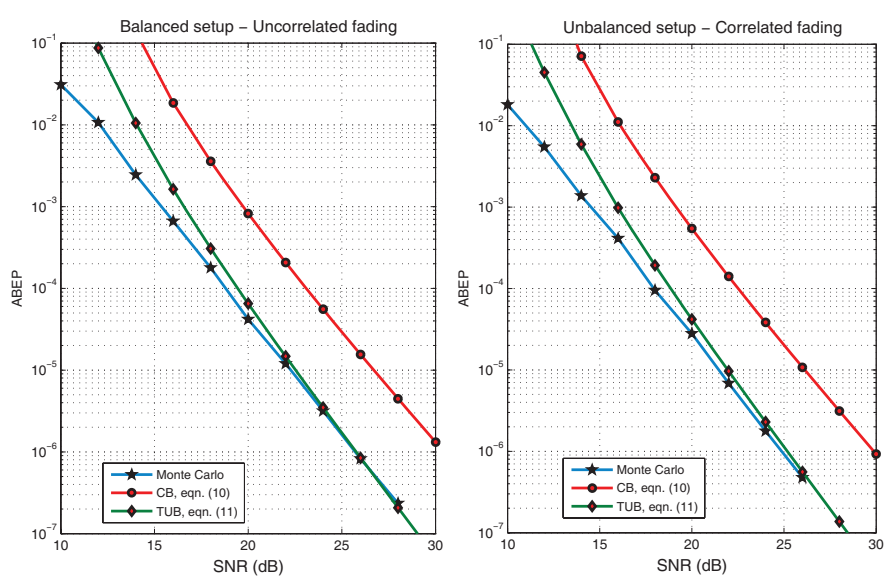

Fig. 7. ABEP for a $4 \times 1$ MISO system: simulation vs. bounds.

substantiate the analytical frameworks introduced in this paper, and confirm that they can be used for the accurate analysis and optimization of TCSM over correlated fading channels.

\section{B. Simulation results}

In this subsection, Monte Carlo simulation results for at least $10^{6}$ channel realizations have been obtained and the 
average BER is plotted versus the average SNR at each receiver input. In all simulations with Rician fading, the Rician $K$ factor is set to $K=3$. This value is within the range of the measured values in indoor communication [42].

The results are grouped in two parts. The first part uses suboptimum receiver since it uses a hard decision Viterbi decoder. The second part, however, is an optimum configuration that uses TuCM [43], [44]. For all systems, two non-systematic convolutional encoders (NSC) with the UMTS interleaving pattern [45] are considered. The receiver uses the Log-MAP algorithm [43, Section 7.8.3] and six turbo decoding iterations to decode the LLR bits.

1) Performance with hard decision Viterbi decoder: In the first result, depicted in Fig. 8, TCSM performance under ideal channel conditions is compared to SM, SCBLAST, and Alamouti. All systems achieve similar spectral efficiency of $4 \mathrm{~b} / \mathrm{s} / \mathrm{Hz}$. TCSM transmits an 8QAM symbol from four transmit antennas to achieve the required spectral efficiency. The considered 8QAM constellation diagram is given in [46, Fig. 5.2-15(a)]. SM is uncoded system and it transmits 4QAM symbol from four transmit antennas. SCBLAST uses the rate $1 / 2$ TCM encoder and transmits 4QAM symbols from four transmit antennas. Finally, Alamouti transmits 32QAM and uses the rate $4 / 5 \mathrm{TCM}$ encoder to achieve the $4 \mathrm{~b} / \mathrm{s} / \mathrm{Hz}$ spectral efficiency.

In ideal channel conditions (the channel paths are uncorrelated), uncoded SM system outperforms all other systems. Similar results are reported in [8], [14]. SM outperforms the proposed TCSM system by about $3 \mathrm{~dB}$. The reason for this behavior is that SM uses low order modulation as compared to TCSM and the TCM coding gain and the set partitioning of the transmit antennas, in TCSM, has no advantage since all channel paths are uncorrelated. However, the situation is different if correlated channel paths are considered, i.e. when Rician fading and SC channels are considered as it is shown later.

In the same figure, Fig. 8, TCSM and SCBLAST outperform each other in a range of SNRs. The BER curves intersects at $11 \mathrm{~dB}$ SNR. The channel coding gain and the lower order modulation in SCBLAST are the reasons for the enhancements at high SNR. In addition, and as discussed previously, the TCM coding and set partitioning add no enhancements to TCSM as the channel paths are uncorrelated in this scenario. Both TCSM and SCBLAST outperform Alamouti scheme by about $2-4 \mathrm{~dB}$. This can be explained due to the use of higher modulation order in Alamouti to achieve the same spectral efficiency as in TCSM and SCBLAST. This also explains the larger SCBLAST gain over TCSM at high SNR. The enhancement of TCSM over SCBLAST at low SNR is not related to the TCM coding and set partitioning. It is mainly due to the underlying working mechanism of SM and the fact that it completely avoids ICI at the receiver side [8].

The advantage of TCSM over all other schemes is obvious from Figs. 9 and 10. In Fig. 9, the performance of all systems in Rician fading channel are depicted. TCSM outperforms SM by $1 \mathrm{~dB}$, SCBLAST by $2 \mathrm{~dB}$, and Alamouti by $4 \mathrm{~dB}$ at a BER of $10^{-4}$. In addition, the gain in SNR at the same BER increases for SC channel as shown in Fig. 10. In this case TCSM outperforms SM by $2 \mathrm{~dB}$, SCBLAST by $4 \mathrm{~dB}$,

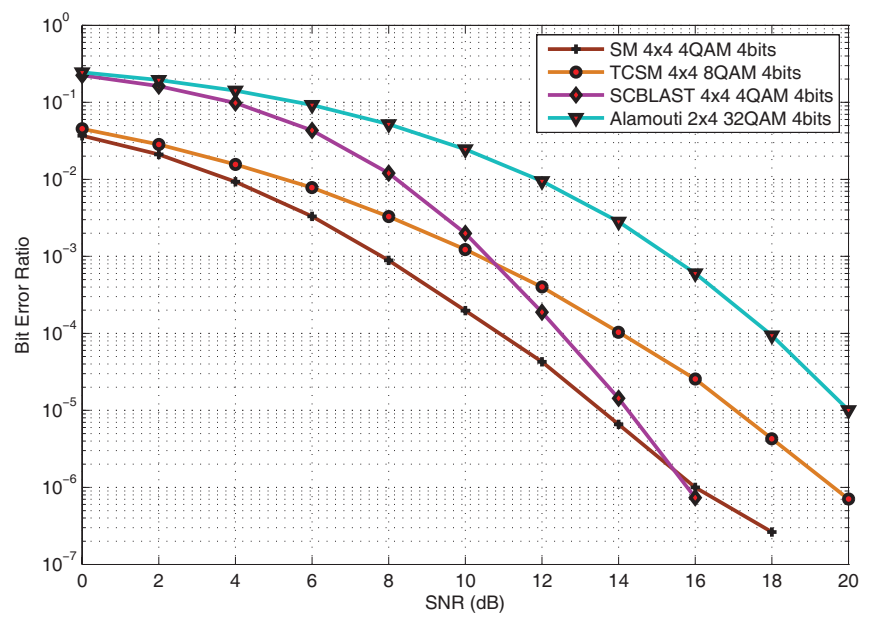

Fig. 8. Performance comparison of SM 4x4 BPSK and TCSM 4x4 4QAM for ideal channel.

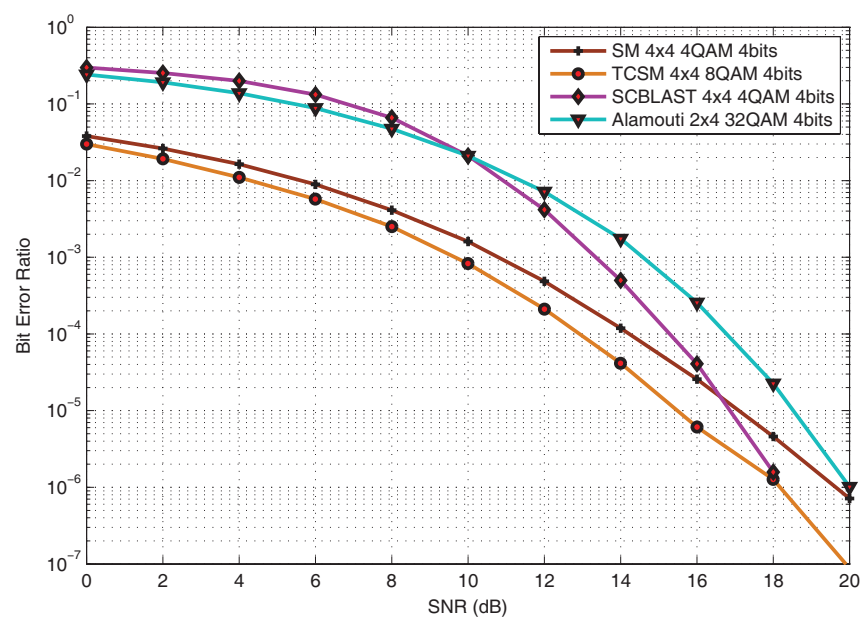

Fig. 9. Performance comparison of SM 4x4 BPSK and TCSM 4x4 4QAM in Rician fading channel with Rician $K$-factor of 3 .

and Alamouti by $5 \mathrm{~dB}$. The significant gains in the presence of channel correlations due to Rician fading or SC can be attributed to TCM encoding and the underlying partitioning of the transmit antennas. The fact that the transmit antennas with larger separation distance are grouped in one set, reduces the effect of correlation and results in a better performance. In other words, the trellis transitions do not allow for an error to occur between antenna one and two or three and four. The errors could only be between one and three or two and four. Hence, the spatial separation between neighbor antennas increases, which leads to better performance in correlated channel conditions.

A significant notice from Fig. 9 is the performance enhancement of Alamouti and TCSM in the presence of Rician fading channel as compared to ideal channel condition. The orthogonality of the Alamouti code is not affected by the presence of LOS component and instead, it gains from the higher SNR values due to the presence of LOS [38]. TCSM demonstrates similar behavior to Alamouti in the presence of LOS path between the transmitter and the receiver and gains $1 \mathrm{~dB}$ in SNR at a BER of $10^{-4}$ as compared to ideal channel condition. This can be explained by the fact that Rician fading increases 


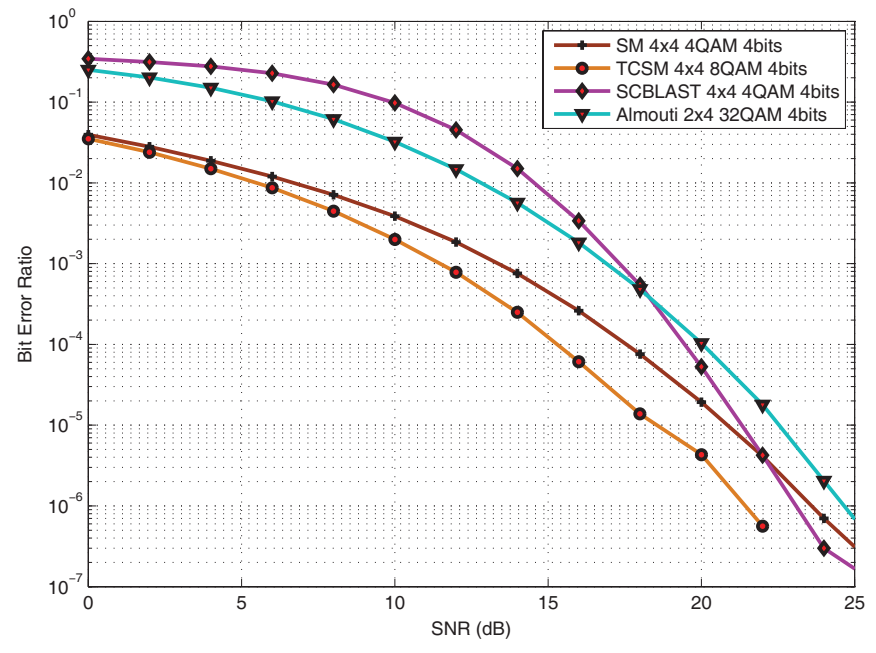

Fig. 10. Performance comparison of SM 4x4 BPSK and TCSM 4x4 4QAM in SC channel with transmit antenna element spacing of $0.1 \lambda$ at the transmitter and $0.5 \lambda$ at the receiver.

the SNR at the receiver side and the underlying set partitioning together with TCM coding eliminates the correlation between transmit antennas. Nonetheless, it should be mentioned that the performance of TCSM in Rician fading channels depends on the number of transmit antennas, the considered modulation order, and the Rician $K$-factor. Different parameters will result in different performance.

In summary, the basic idea of the proposed TCSM scheme is to divide the existing antennas into sets using TCM such that each set maximizes the spatial distance between its antennas, and therefore minimizes the effect of correlation fading.

2) Performance with turbo coded modulation (TuCM): All schemes use two parallel concatenated rate $1 / 2$ NSC with the following generator polynomials, $G_{1}=\left[\begin{array}{cc}1 & \frac{1+D^{2}}{1+D}\end{array}\right]$ and $G_{2}=\left[\begin{array}{cc}1 & \frac{1+D^{2}}{D}\end{array}\right]$. A spectral efficiency of $1 \mathrm{~b} / \mathrm{s} / \mathrm{Hz}$ is achieved for all systems. In Alamouti scheme, the four output bits for each incoming data bit are mapped to 16QAM symbol and transmitted from the two transmit antennas. In SCBLAST, the output bits are mapped to BPSK and transmitted from the four transmit antennas. At the receiver, SCBLAST uses list sphere decoder (LSD) algorithm [47], [48] to create the LLR values to be decoded by the turbo decoder. For TCSM, the LLRs are calculated as in subsection II-B. TCSM maps two bits to antenna indices and two bits to signal symbol to achieve the spectral efficiency of $1 \mathrm{~b} / \mathrm{s} / \mathrm{Hz}$. The calculated LLR values are processed by the turbo decoder and the BER is calculated.

Simulation results for TCSM, Alamouti, and SCBLAST in ideal, Rician, and SC channel conditions with the above discussed TuCM are depicted in Fig. 11. The performance of all systems demonstrate similar trend as discussed in the previous subsection. Rician fading channel enhances the performance of TCSM and Alamouti. TCSM demonstrate the best performance and in ideal channel conditions outperforms Alamouti by about $3 \mathrm{~dB}$ and SCBLAST by about $4 \mathrm{~dB}$. Larger gains are achieved in Rician and SC channel conditions. SCBLAST greatly suffers from the presence of non idealistic channel conditions and as compared to ideal channel conditions, Rician fading and SC degrade the performance by about

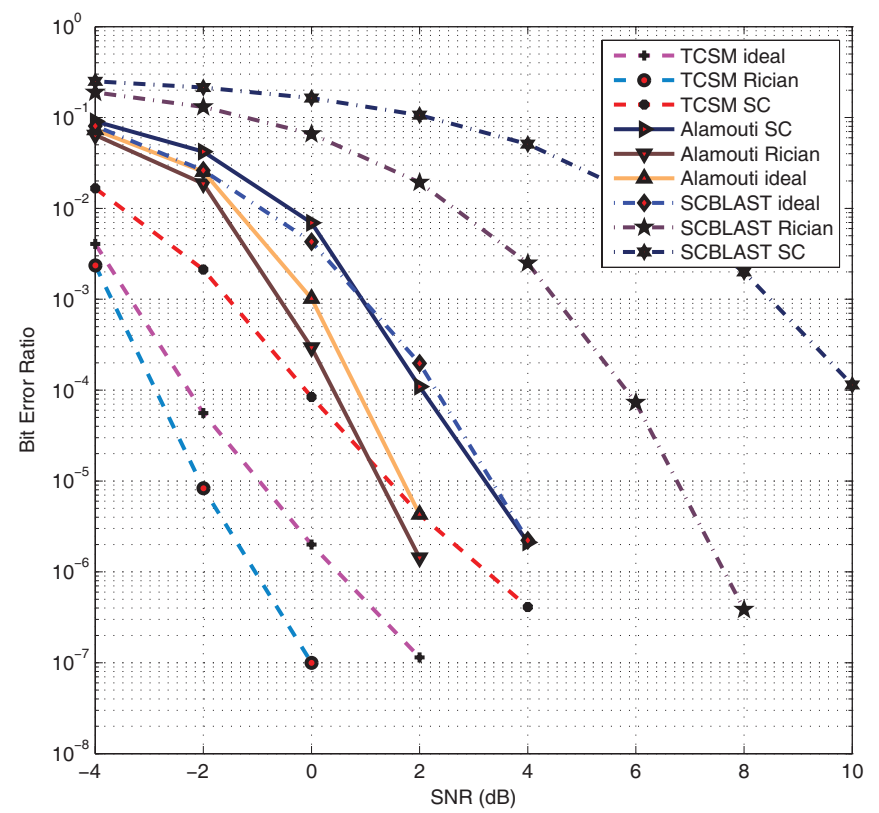

Fig. 11. Turbo coded performance comparison of TCSM 4x4 4QAM, SCBLAST 4x4 4QAM, and Alamouti 2x4 16QAM in ideal, SC, and Rician channel conditions. Each scheme uses two parallel concatenated rate 1/2 NSC. The receiver uses six turbo decoding iterations to decode the LLR bits.

$4 \mathrm{~dB}$ and $9 \mathrm{~dB}$, respectively.

\section{TCSM, SCBLAST, AND ALAMOUTI RECEIVER COMPLEXITY COMPARISON}

In this section, the receiver complexity of SCBLAST, Alamouti, and TCSM is studied. It is assumed that only multiplications and additions of complex numbers are an arithmetic operation. It is also assumed that channel coding and Viterbi decoding consumes the same number of complex operations in all systems and ignored in the following.

The required number of complex operations to detect the transmit antenna index and the transmitted symbol in TCSM is calculated in [14] and shown to be $2 N_{t} N_{r}+N_{t} M+M$.

The V-BLAST SD complexity with adaptive radius is calculated in [49] and shown to require

$$
\sum_{k=1}^{N_{t}} \frac{M k \pi^{\frac{k}{2}}}{\Gamma\left(\frac{k}{2}+1\right)} C^{k}+2 N_{t}^{2}
$$

complex multiplications and

$$
\sum_{k=1}^{N_{t}} \frac{M(k+1) \pi^{\frac{k}{2}}}{\Gamma\left(\frac{k}{2}+1\right)} C^{k}+2 N_{t}^{2}-N_{t}+2
$$

complex additions; where $\Gamma$ is the gamma function and $C$ is the sphere radius. The sphere radius, as considered in this paper, is a function of the SNR and the used modulation order given as [50],

$$
C^{2}=2 \delta N_{r} \sigma_{n}^{2}
$$

where $\delta$ is a constant commonly defined based on experience and a value of 3 is considered here [50].

Alamouti detection process requires $N_{r}^{2}$ complex multiplications and $2\left(2 N_{r}-1\right)$ complex additions for two transmitted symbols. Therefore, a total of $0.5\left(N_{r}^{2}+4 N_{r}-2\right)$ complex 
TABLE I

COMPLEXITY COMPARISON BETWEEN TCSM, SCBLAST, AND Alamouti

\begin{tabular}{|c|c|c|c|c|}
\hline \multicolumn{5}{|c|}{ Complexity Comparison } \\
\hline \hline Alamouti & TCSM & \multicolumn{3}{|c|}{ SCBLAST $(4 \times 4$ 4QAM) } \\
\hline 2x4 32QAM & $4 \times 48$ QAM & SNR $=0 \mathrm{~dB}$ & SNR=10 dB & SNR=20 dB \\
\hline 15 & 192 & 950 & 466 & 314 \\
\hline \hline
\end{tabular}

operations are required at the receiver for the detection of one transmitted symbol [8].

For the considered systems in this paper, the required number of complex operations are listed in Table I. The considered SCBLAST complexity equations are calculated for three different SNR values, $0 \mathrm{~dB}, 10 \mathrm{~dB}$, and $20 \mathrm{~dB}$.

The proposed TCSM requires moderate number of complex operations which lie between the complexity of SCBLAST and Alamouti. For instance, at $0 \mathrm{~dB}$ SNR, TCSM requires $80 \%$ less complexity as compared to SCBLAST. Alamouti scheme is shown to have the least complexity but an inferior performance as compared to TCSM. SCBLAST on the other hand requires the highest number of complex operations and, further its performance degrades severely in the presence of channel imperfections. However, if high data rate is required and large number of transmit antennas are available, then, SCBLAST is better suitable provided that channel correlations are moderate.

\section{CONCLUSION}

TCM concept is adopted in a novel way in this paper to combat performance degradation of SM in correlated channel conditions. The main idea is to divide the transmit antennas into sets with each set having the maximum possible separation distance between its antennas. As a result, the correlation between the channel paths of each set is reduced. Two analytical bounds for performance analysis of TCSM over correlated Rayleigh fading channels are proposed in this paper. The comparison with Monte Carlo simulation demonstrated good agreement with the analytical framework. In addition, the performance of TCSM has been simulated in several channel conditions and compared to other MIMO systems. It has been shown that a major enhancements in SNR can be obtained in Rician fading and correlated channel conditions. As compared to Alamouti and V-BLAST, significant enhancements in BER are reported. In addition, the complexity of the proposed technique is shown to be much lower than V-BLAST and higher than Alamouti. The achieved performance and complexity of TCSM makes it suitable candidate for future wireless MIMO systems. TCSM is shown to perform better in the presence of LOS conditions. Moreover, TCSM allows for low spatial separation between the transmit antennas without significant performance degradation. The performance of the proposed idea is shown to be further enhanced by considering turbo codes and channel coding of the transmitted symbols. In addition, the detection algorithm of TCSM might be further optimized and techniques such as sphere decoder could be used to reduce its complexity. This, however, is subject to future works.

\section{APPENDIX \\ DERIVATION OF (18)}

The PEP given in (18) can be obtained by using the following analytical derivations. The RVs $h_{\kappa}$ and $h_{\nu}$ in (17) are distributed according to a Rayleigh distribution and are explicitly written in Section IV as follows:

$$
\left\{\begin{array}{l}
h_{\nu}=h_{\nu}^{\mathrm{R}}+j h_{\nu}^{\mathrm{I}} \\
h_{\kappa}=h_{\kappa}^{\mathrm{R}}+j h_{\kappa}^{\mathrm{I}}
\end{array}\right.
$$

Accordingly, (17) can be re-written as follows:

$$
\begin{aligned}
\operatorname{PEP} & \left(\kappa \rightarrow \nu \mid h_{\kappa}, h_{\nu}\right) \\
& =Q\left(\sqrt{\bar{\gamma}\left|\left(h_{\kappa}^{\mathrm{R}}-h_{\nu}^{\mathrm{R}}\right)+j\left(h_{\kappa}^{\mathrm{I}}-h_{\nu}^{\mathrm{I}}\right)\right|^{2}}\right) \\
& =Q\left(\sqrt{\bar{\gamma}\left|X_{\kappa, \nu}+j Y_{\kappa, \nu}\right|^{2}}\right)=Q\left(\sqrt{\bar{\gamma} \chi_{\kappa, \nu}}\right)
\end{aligned}
$$

where $X_{\kappa, \nu}=h_{\kappa}^{\mathrm{R}}-h_{\nu}^{\mathrm{R}}, Y_{\kappa, \nu}=h_{\kappa}^{\mathrm{I}}-h_{\nu}^{\mathrm{I}}$, and $\chi_{\kappa, \nu}=$ $\left|X_{\kappa, \nu}+j Y_{\kappa, \nu}\right|^{2}$.

In the light of the correlation model described in Section IV, the RVs $X_{\kappa, \nu}$ and $Y_{\kappa, \nu}$ are distributed according to a normal distribution and are uncorrelated. In particular, $X_{\kappa, \nu} \sim \mathcal{N}\left(0, \bar{\sigma}_{\nu, \kappa}^{2}\right)$ and $Y_{\kappa, \nu} \sim \mathcal{N}\left(0, \bar{\sigma}_{\nu, \kappa}^{2}\right)$ with $\bar{\sigma}_{\nu, \kappa}^{2}=$ $\sigma_{\nu}^{2}+\sigma_{\kappa}^{2}-2 \varphi_{\nu, \kappa} \sigma_{\nu} \sigma_{k}$.

Accordingly, from [46, eqn. (2.1.127)] it follows that the RV $\tilde{\chi}_{\kappa, \nu}=\sqrt{\chi_{\kappa, \nu}}$ is distributed according to a Rayleigh distribution with $\mathrm{E}\left\{\tilde{\chi}_{\kappa, \nu}^{2}\right\}=2 \bar{\sigma}_{\nu, \kappa}^{2}$ [46, eqn. (2.1.130)]. Moving from these considerations, (18) follows from [46, eqn. (14.3.7)].

\section{REFERENCES}

[1] G. Ungerboeck, "Channel coding with multilevel/phase signals," IEEE J. Inf. Technol., vol. 28, no. 1, pp. 55-67, 1982.

[2] E. Telatar, "Capacity of multi-antenna Gaussian channels," European Trans. Telecommun., vol. 10, no. 6, pp. 585-595, Nov./Dec. 1999.

[3] S. M. Alamouti, "A simple transmit diversity technique for wireless communications," IEEE J. Sel. Areas Commun., vol. 16, no. 8, pp. 14511458, Oct. 1998.

[4] V. Tarokh, H. Jafarkhani, and A. Calderbank, "Space-time block codes from orthogonal designs," IEEE Trans. Inf. Theory, vol. 45, no. 5, pp. 1456-1467, July 1999.

[5] G. J. Foschini, "Layered space-time architecture for wireless communication in a fading environment when using multi-element antennas," Bell Labs Technical J., vol. 1, no. 2, pp. 41-59, Sep. 1996.

[6] P. Wolniansky, G. Foschini, G. Golden, and R. Valenzuela, "V-BLAST: an architecture for realizing very high data rates over the rich-scattering wireless channel," in Proc. Unino Radio-Scientifique Internationale (URSI) Intern. Symp. on Signals, Systems, and Electronics (ISSSE), Sep. 1998, pp. 295-300.

[7] M. Sellathurai and S. Haykin, "Turbo-BLAST for wireless communications: theory and experiments," IEEE Trans. Signal Process., vol. 50, no. 10, pp. 2538-2546, Oct. 2002.

[8] R. Mesleh, H. Haas, S. Sinanović, C. W. Ahn, and S. Yun, "Spatial modulation," IEEE Trans. Veh. Technol., vol. 57, no. 4, pp. 2228-2241, July 2008.

[9] S. Ganesan, R. Mesleh, H. Haas, C. W. Ahn, and S. Yun, "On the performance of spatial modulation OFDM," in Proc. Asilomar Conference on Signals, Systems, and Computers, Pacific Grove, CA, USA, Oct. 2006, pp. 1825-1829.

[10] E. Viterbo and J. Boutros, "A universal lattice code decoder for fading channels," IEEE Trans. Inf. Theory, vol. 45, no. 5, pp. 1639-1642, July 1999.

[11] O. Damen, A. Chkeif, and J.-C. Belfiore, "Lattice code decoder for space-time codes," IEEE Commun. Lett., vol. 4, no. 5, pp. 161-163, May 2000. 
[12] S. M. Alamouti, V. Tarokh, and P. Poon, "Trellis-coded modulation and transmit diversity: design criteria and performance evaluation," in Proc. IEEE 1998 International Conference on Universal Personal Communications ICUPC '98, vol. 1, Florence, Italy, Oct. 1998, pp. 703707.

[13] J. Jeganathan, A. Ghrayeb, L. Szczecinski, and A. Ceron, "Space shift keying modulation for MIMO channels," IEEE Trans. Wireless Commun., vol. 8, no. 7, pp. 3692-3703, July 2009.

[14] J. Jeganathan, A. Ghrayeb, and L. Szczecinski, "Spatial modulation: optimal detection and performance analysis," IEEE Commun. Lett., vol. 12, no. 8, pp. 545-547, Aug. 2008.

[15] A. Paulraj, R. Nabar, and D. Gore, Introduction to Space-Time Wireless Communications. Cambridge University Press, 2003.

[16] A. Forenza, D. Love, and R. Heath Jr., "A low complexity algorithm to simulate the spatial covariance matrix for clustered MIMO channel models," in Proc. IEEE Vehicular Technology Conference (VTC 2004Fall), vol. 2, Los Angeles, CA, USA, May 2004, pp. 889-893.

[17] R. Mesleh, "Spatial modulation: a spatial multiplexing technique for efficient wireless data transmission," Ph.D. dissertation, Jacobs University, Bremen, Germany, June 2007.

[18] Q. Spencer, B. Jeffs, M. Jensen, and A. Swindlehurst, "Modeling the statistical time and angle of arrival characteristics of an indoor multipath channel," IEEE J. Sel. Areas Commun., vol. 18, no. 3, pp. 347-360, Mar. 2000.

[19] V. Erceg et al., TGn Channel Models, IEEE P802.11 Wireless LANs, IEEE Std. IEEE 802.11-03/940r4, May 10, 2004. Retrieved Jan. 12, 2007 from http://www.nari.ee.ethz.ch/ dsbaum/11-03-0940-04-000n-tgnchannel-models.pdf.

[20] G. T. S. Group, Spatial Channel Model, Spatial Channel Model AHG (combined ad-hoc from 3GPP and 3GPP2), 3GPP Std. SCM-134 text V6.0, Apr. 22, 2003.

[21] A. Hottinen, O. Tirkkonen, and R. Wichman, Multi-Antenna Tansceiver Techniques for $3 G$ and Beyond. John Wiley \& Sons Ltd., 2003.

[22] A. Viterbi, "Convolutional codes and their performance in communication systems," IEEE Trans. Commun., vol. 19, no. 5, pp. 751-772, Oct. 1971.

[23] S. Haykin, Communication Systems, 4th ed. Wiley, 2000.

[24] M. K. Simon and M.-S. Alouini, Digital Communication over Fading Channels: A Unified Approach to Performance Analysis, 1st ed. John Wiley \& Sons, Inc., 2000.

[25] M. Di Renzo and H. Haas, "A general framework for performance analysis of space shift keying (SSK) modulation for MISO correlated Nakagami-m fading channels," IEEE Trans. Commun., to appear.

[26] M. Di Renzo and H. Haas, "On the performance of space shift keying MIMO systems over correlated Rician fading channels," in Proc. ITG International Workshop on Smart Antennas (WSA 2010), Bremen, Germany, Feb. 2010.

[27] G. Foschini, D. Chizhik, M. Gans, C. Papadias, and R. Valenzuela, "Analysis and performance of some basic space-time architectures," IEEE J. Sel. Areas Commun., vol. 21, no. 3, pp. 303-320, Apr. 2003.

[28] T. M. Duman and A. Ghrayeb, Coding for MIMO Communication Systems. John Wiley and Sons, 2007.

[29] B. Hochwald and S. ten Brink, "Achieving near-capacity on a multipleantenna channel," IEEE Trans. Commun., vol. 51, no. 3, pp. 389-399, Mar. 2003.

[30] A. Chan and I. Lee, "A new reduced-complexity sphere decoder for multiple antenna systems," in Proc. IEEE International Conference on Communications (ICC'02), vol. 1, New York, USA, Apr. 2002, pp. 460464.

[31] M. Damen, K. Abed-Meraim, and M. Lemdani, "Further results on the sphere decoder," in Proc. IEEE International Symposium on Information Theory, Washington, DC, June 2001, pp. 333.

[32] A. Burg, M. Borgmann, M. Wenk, M. Zellweger, W. Fichtner, and H. Bolcskei, "VLSI implementation of MIMO detection using the sphere decoding algorithm," IEEE J. Solid-State Circuits, vol. 40, no. 7, pp. 1566-1577, July 2005.

[33] L. Barbero and J. Thompson, "Extending a fixed-complexity sphere decoder to obtain likelihood information for turbo-MIMO systems," IEEE Trans. Veh. Technol., vol. 57, no. 5, pp. 2804-2814, Sep. 2008.

[34] _ , "FPGA design considerations in the implementation of a fixedthroughput sphere decoder for MIMO systems," in Proc. International Conference on Field Programmable Logic and Applications FPL '06, Madrid, Spain, Aug. 2006, pp. 1-6.

[35] 3rd Generation Partnership Project (3GPP), Technical Specification Group Radio Access Network, Summary of MIMO Schemes for EUTRA, 3GPP Std. R1-051 238.
[36] S. N. Diggavi, N. Al-Dhahir, A. Stamoulis, and A. R. Calderbank, "Great expectations: the value of spatial diversity in wireless networks," Proc. IEEE, vol. 92, no. 2, pp. 219-270, Feb. 2004.

[37] S. Parker, M. Sandell, and M. Lee, "The performance of space-time codes in office environments," in Proc. VTC 2003-Spring Vehicular Technology Conference, vol. 1, Jeju Island, Korea, Apr. 2003, pp. 741745.

[38] H. El Gamal, "On the robustness of space-time coding," IEEE Trans. Signal Process., vol. 50, no. 10, pp. 2417-2428, Oct. 2002.

[39] J. G. Andrews, A. Ghosh, and R. Muhamed, Fundamentals of WiMAX. Pearson Education, Inc., 2007.

[40] H. Jafarkhani, Space-Time Coding: Theory and Practice. Cambridge University Press, 2005.

[41] Y. A. Chau and S.-H. Yu, "Space modulation on wireless fading channels," in Proc. VTC 2001 Fall Vehicular Technology Conference IEEE VTS 54th, Atlantic City, New Jersey, USA, vol. 3, Oct. 2001, pp. $1668-1671$.

[42] M. Carroll and T. Wysocki, "Fading characteristics for indoor wireless channels at 5GHr unlicensed bands," in Proc. IEEE Joint First Workshop on Mobile Future and Symposium on Trends in Communications (SympoTIC'03), Bratislava, Slovakia, Oct. 2003, pp. 102-105.

[43] R. H. Morelos-Zaragoza, The Art of Error Correcting Coding. John Wiley \& Sons Ltd., 2006.

[44] A. Stefanov and T. M. Duman, "Turbo-coded modulation for systems with transmit and receive antenna diversity over block fading channels: system model, decoding approaches, and practical considerations," IEEE J. Sel. Areas Commun., vol. 19, no. 5, pp. 958-968, May 2001.

[45] ETSI TS 125212 V8.5.0, "Multiplexing and channel coding (FDD)," Sophia Antipolis Valbonne, France, 2009.

[46] J. G. Proakis, Digital Communications, 4th ed., ser. McGraw-Hill Series in Electrical and Computer Engineering, S. W. Director, Ed. McGrawHill Higher Education, Dec. 2000.

[47] B. M. Hochwald and S. ten Brink, "Achieving near-capacity on a multiple-antenna channel," IEEE Trans. Commun., vol. 51, no. 3, pp. 389-399, Mar. 2003.

[48] X. Wang, K. Niu, Z. He, W. Wu, and X. Zhang, "List sphere decoding combined with linear detection-based iterative soft interference cancellation via exit chart," in Proc. IEEE 17th International Symposium on Personal, Indoor and Mobile Radio Communications (PIMRC), Athens, Greece, Sep. 2006, pp. 1-5.

[49] B. Hassibi and H. Vikalo, "On the sphere-decoding algorithm I: expected complexity," IEEE Trans. Signal Process., vol. 53, no. 8, pp. 2806-2818, Aug. 2005.

[50] X. Xia, H. Hu, and H. Wang, "Reduced initial searching radius for sphere decoder," in Proc. IEEE 18th International Symposium on Personal, Indoor and Mobile Radio Communications PIMRC 2007, Athens, Greece, Sep. 2007, pp. 1-4.

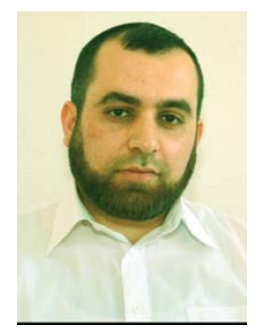

Raed Mesleh (S'00-M'08) received the B.Sc. degree in communication engineering from Yarmouk University, Irbid, Jordan in 2000, the M.Sc. degree in communication technology from Ulm University, Ulm, Germany in 2004 and the Ph.D. degree in electrical engineering from the Jacobs University, Bremen, Germany in 2007. Since September 2007, he has been with the School of Electrical Engineering and Computer Science, Jacobs University, Bremen, Germany where he currently works as a postdoctoral fellow. His main research interests are in wireless communication and optical wireless communication with particular focus on MIMO techniques. He is a co-inventor of seven patents, three of them are already granted, in the field of RF and optical wireless communication systems.

Dr. Mesleh received a tuition waiver for his Ph.D. study at Jacobs University along with a three years scholarship. In 2003, he received the award for the best student performance in the M.Sc. course at Ulm University (LEG award) and, in 2000, he received the award for the best graduate communication engineer in year 2000 from Yarmouk University. Dr. Mesleh joined Orange in Amman, Jordan (2000-2001), as a system Engineer and worked as a consultant for NOKIA in Saudi Arabia from 2001 to 2002. During his M.Sc. study (20022004), he partly worked with LogicaCMG as a protocol and testing engineer. Dr. Mesleh serves as a technical reviewer for several IEEE transaction journals and international conferences and he is a TPC member for several international conferences in wireless communications. 


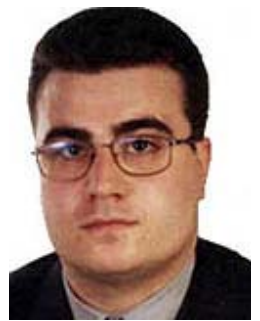

Marco Di Renzo (SM'05-AM'07-M'09) was born in L'Aquila, Italy, in 1978. He received the Laurea (cum laude) and the Ph.D. degrees in Electrical and Information Engineering from the Department of Electrical and Information Engineering, University of L'Aquila, Italy, in April 2003 and January 2007, respectively. Since January 2010 , he has been a Researcher ("Chargé de Recherche") with the French National Center for Scientific Research (CNRS), and a research staff member of the Laboratory of Signals and Systems (L2S), a joint research laboratory of the CNRS, the École Supérieure d'Électricité (SUPÉLEC), and the University of Paris-Sud XI, Paris, France. His main research interests are in the area of wireless communication theory, signal processing, and information theory.

Dr. Di Renzo was awarded a special mention for the outstanding five-year (1997-2003) academic career from the University of L'Aquila, Italy, and a personal "Torres Quevedo" Grant (PTQ-08-01-06437) from the "Ministry of Science and Innovation" in Spain for his research on ultra wide band systems and cooperative localization for wireless sensor networks.

Dr. Di Renzo is a Member of the IEEE and IEEE Communications Society, and serves as reviewer for transaction journals and international conferences. He served as Publicity Chair of the 2010 International Conference on Mobile Lightweight Wireless Systems (Mobilight). He also serves as Technical Program Committee (TPC) member and Session Chairman of several international conferences in communication.

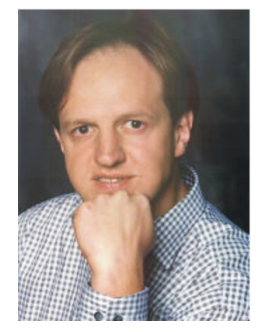

Harald Haas (S'98-A'00-M'03) received the $\mathrm{PhD}$ degree from the University of Edinburgh in 2001. From 2001 to 2002, he was project manager at Siemens AG for an international research project. He joined International University Bremen (Germany), now Jacobs University Bremen, in September 2002 where he has since been Associate Professor of Electrical Engineering. In June 2007, he joined the University of Edinburgh (Scotland/UK) where he is Reader in the Institute for Digital Communications (IDCOM). His main research interests are in radio resource and interference management, wireless system design and analysis, MIMO techniques and optical wireless communications. Haas received a best paper award at the International Symposium on Personal, Indoor and Mobile Radio Communications (PIMRC) in Osaka/Japan in 1999 and holds more than 15 patents in the area of wireless communications. He contributed a chapter to the Handbook of Information Security entitled "Air Interface Requirements for Mobile Data Services" by John Wiley \& Sons, Inc. He co-authored a book entitled Next Generation Mobile Access Technologies: Implementing $T D D$ with Cambridge University Press. This textbook is now being translated into Chinese by the publisher. His work on optical wireless communication was selected for publication in "100 Produkte der Zukunft (100 Products of the Future)" authored by Nobel Laureate T. W. Hänsch. Since 2007 he is a Regular High Level Visiting Scientist supported by the Chinese "111 program" at Beijing University of Posts and Telecommunications (BUPT).

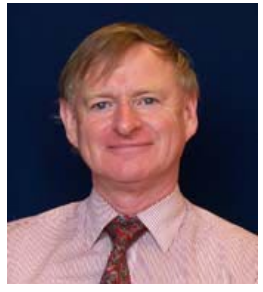

Peter M. Grant (M'77-SM'83-F'96) was born in St. Andrews, Scotland. He received the B.Sc. degree in electronic engineering from the Heriot-Watt University, Edinburgh, Scotland, in 1966, the Ph.D. degree from the University of Edinburgh in 1975 and honorary DEng's from the Heriot-Watt in 2006 and from Napier University in 2007.

$\mathrm{He}$ worked in radio communications for the Plessey Company, before he was appointed to a research fellowship at the University of Edinburgh. He was subsequently promoted through to a full professor of Electronic Signal Processing and on to Departmental chair. He served from 2002-2008 as head of the School of Engineering and Electronics at Edinburgh.

During academic year 1977-78, he was a visiting professor at the Ginzton Laboratory, Stanford University, and in 1985-86 he was a visiting staff member at the MIT Lincoln Laboratory. He was awarded the 82nd (2004) Faraday Medal by the Institution of Electrical Engineers (IEE) for his work on CDMA receiver designs and adaptive filters. In 1974 and again in 1977 he was awarded the Bulgin premium from the then Institution of Electronic and Radio Engineers and in 1982 their Lord Mountbatten premium. In 1994 he was awarded both the IEE Marconi and Langham Thompson premia.

Professor Grant was president of EURASIP in 2000-2002, he was chairman of EUSIPCO-94 and technical programme chairman for ICASSP-89. In 1998 he was appointed by the IEEE Signal Processing Society as a distinguished lecturer on DSP for Mobile Communications. He served from 1980-1996 as an honorary editor of IEE PROCEEDINGS title "Vision Image and Signal Processing." He was a member in 1992 and 1996 and was chair of the 2001 Universities Funding Council research assessment panel for the UK Electrical Engineering Departments. He served as a director of the Mobile VCE from 2007-9.

Professor Grant was in 2007 appointed to be the 8th Regius Professor of Engineering at The University of Edinburgh. In 2009 he was made an officer of the order of the British Empire (OBE) in the Queen's birthday honours list. He holds fellowships of the IEEE, IET, Royal Academy of Engineering and the Royal Society of Edinburgh. 